acidemia, $13-\mathrm{OH}$-metilglutaric aciduria, 1 glutaric aciduria type 1, 1 metilglutaconic acidemia, 1 trimethylaminuria, 1 propionic acidemia, 2 tyrosinemia and 3 homocystinuria. Ventilatory support needed in $13 / 20$, vasoactive agents $11 / 20$, peritoneal dialysis $8 / 20$, continuous veno-venous haemofiltration $4 / 20$. 8 patients died; among survivors, $2 / 12$ madurative failure, $3 / 12$ serious neurological sequelae, $1 / 12$ liver failure, needing transplantation.

Conclusions Aminoacidopathies diagnosed by Ms/Ms start early with treatment. Wide range of presentation symptoms and findings.

\section{THE NIEMANN-PICK TYPE C SUSPICION INDEX TOOL: EXAMINATION OF ITS DISCRIMINATORY POWER BY AGE AND ASSOCIATIONS BY LEADING SYMPTOMS}

doi:10.1136/archdischild-2012-302724.1032

${ }^{1} \mathrm{CJ}$ Hendriksz, ${ }^{2} \mathrm{M}$ Pineda, ${ }^{3} \mathrm{FA}$ Wijburg, ${ }^{4} \mathrm{~F}$ Sedel, ${ }^{5} \mathrm{M}$ Fahey, ${ }^{6} \mathrm{M}$ Walterfang, ${ }^{7} \mathrm{MC}$ Patterson, ${ }^{8} \mathrm{H}$ Chadha-Boreham, ${ }^{8} \mathrm{SA}$ Kolb, ${ }^{9} \mathrm{JE}$ Wraith. 'Department of Adult Inherited Metabolic Disease, Salford Royal Hospital NHS Foundation Trust, Manchester, UK; ${ }^{2}$ Department of Pediatric Neurology, Hospital Sant Joan de Déu, Barcelona, Spain; ${ }^{3}$ Department of Paediatrics, University of Amsterdam, Amsterdam, The Netherlands,

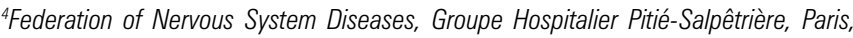
France; ${ }^{5}$ Department of Paediatrics, Monash University; ${ }^{6}$ Department of Neuropsychiatry, University of Melbourne, Melbourne, VIC, Australia; 'Division of Child and Adolescent Neurology, Mayo Clinic, Rochester, MN, USA; ${ }^{8}$ Actelion Pharmaceuticals Ltd., Allschwil, Switzerland: 'Willink Biochemical Genetics Unit, Royal Manchester Children's Hospital, Manchester, UK

Background and Aims The Suspicion Index (SI) screening tool was developed to identify suspected patients with Niemann-Pick disease type C (NP-C, Neurology, 2012). The SI provides Risk Prediction Score (RPS) based on NP-C symptoms within and across domains (visceral, neurological, and psychiatric). To further examine a) discriminatory power of the SI by age and b) symptom-associations by NP-C suspicion-level and leading symptoms.

Methods The original retrospective data were split into three age groups, where NP-C positive cases were: $>16$ years $(n=30), 4-16$ years $(n=18)$, and $<4$ years $(n=23)$, and patients' RPS was analysed by logistic regression. Co-occurrence of symptoms within groups of suspicion-level (low, medium, and high) and leading symptoms (presence/absence of ataxia, cognitive decline, psychosis, and splenomegaly) were analysed descriptively.

Results NP-C positive cases vs. controls showed strong discriminatory power of RPS. Area under the Receiver Operating Characteristic curve was 0.964 (>16 years) and 0.981 (4-16 years) but a weaker 0.562 for infants ( $<4$ years). Patients with RPS $<70$ were characterised by a lack of psychiatric symptoms and low levels of neurological involvement, suggestive of a more visceral phenotype. In patients $>4$ years, prominent leading symptoms' associations were: ataxia with "dystonia, dysarthria/dysphagia and cognitive decline"; psychosis with "dysarthria/dysphagia"; and psychotic symptoms with "cognitive decline and treatment-resistant psychiatric symptoms".

Conclusions The SI tool maintains strong discriminatory power in patients $>4$ years but is not as useful for infants $<4$ years. The SI is informative regarding the association and co-occurrence of symptoms in patients with NP-C.

\section{CENTRAL NEUROGENIC HYPERVENTILATION MAY CAUSE METABOLIC ALKALOSIS IN MITOCHONDRIAL ENCEPHALOPATHY}

doi:10.1136/archdischild-2012-302724.1033

${ }^{1} \mathrm{P}$ Toth-Heyn, ${ }^{1} \mathrm{C}$ Lodi, ${ }^{1} \mathrm{~A}$ Jermendy, ${ }^{2} \mathrm{G}$ Rudas. ${ }^{1 /}$ st Department of Pediatrics; ${ }^{2} \mathrm{MRKK}$, Semme/weis University, Budapest, Hungary
Background The differential diagnosis of respiratory alkalosis (RA) includes a state called central neurogenic hyperventilation $(\mathrm{CNH})$. In the few reported cases of $\mathrm{CNH}$ the etiology was a stimulation of the respiratory center by an infiltrative tumor in the cerebral pons. In some cases, a shift in the cerebral $\mathrm{pH}$ to acidic range was also hypothesized.

Case Report We report the case of a six year-old boy with a known Pearson syndrome, a mitochondrial disorder affecting bone marrow, pancreas and renal tubules. He was admitted to our PICU with deteriorating mental status and compensated metabolic acidosis (lactic, hyperchloremic and tubular). On admission, blood gas analysis showed a $\mathrm{pH}$ of 7.30 with a disproportionately low compensating $\mathrm{pCO}_{2}$ of $10 \mathrm{mmHg}\left(\mathrm{HCO}_{3} 4.9 \mathrm{mmol} / \mathrm{L}\right)$. Serum $\mathrm{HCO}_{3}$ normalized by substitution $(21.0 \mathrm{mmol} / \mathrm{L})$, when he developed a $\mathrm{RA}(\mathrm{pH} 7.51$, $\mathrm{pCO}_{2} 24 \mathrm{mmHg}$ ) persisting over 48 hours, even during sleeping periods. After reviewing his previous blood gas results, this phenomenon was present for years. After excluding known etiologies of RA, we suspected $\mathrm{CNH}$ caused by intra-cerebral acidosis. The $\mathrm{pH}$ and $\mathrm{HCO}_{3}$ were lower, while lactate was higher in cerebro-spinal fluid than in serum. An MR spectroscopy confirmed cerebral lactate accumulation, showing a peak in the posterior cerebrum. Encephalopathy is not among the classic manifestations of Pearson syndrome.

Conclusion We were able to demonstrate elevated local lactate level leading to intra-cerebral acidosis, stimulation of the respiratory center and causing long-standing hyperventilation. This phenomenon adds a new aspect to the complex clinical picture of mitochondrial disorders.

\section{A HYPOTONIC INFANT WITH METHYLENE TETRAHYDROFOLATE REDUCTASE (MTHFR) DEFICIENCY; HOMOZYGOUS MUTATION OF C.1015T>G IN MTFHR GENE}

doi:10.1136/archdischild-2012-302724.1034

${ }^{1} Y D$ Hanedar, ${ }^{2} \mathrm{~N}$ Yalindag-Ozturk, ${ }^{3} \mathrm{~N}$ Hekim. ${ }^{1}$ Department of Pediatrics; ${ }^{2}$ Department of Pediatric Critical Care, Marmara University; ${ }^{3}$ Center for Life Sciences and Technology (INOVITA Project), Bogazici University, Istanbul, Turkey

Background Methylene tetrahydrofolate reductase (MTHFR) deficiency is a rare autosomal recessive disorder, caused by mutated alleles of the MTHFR gene. Since this enzyme catalyzes the conversion of 5.10-methylenetetrahydrofolate to 5-methyltetrahydrofolate, its deficiency results in hyperhomocysteinemia, homocystinuria and hypomethionemia. The clinical manifestations vary from asymptomatic to fatal disease with severe neurodevelopmental delay and epileptic encephalopathy.

Case Our patient was a two-month old female born from consanguineous parents presenting with infantile spasms, hypotonia and microcephalus. She was transferred to our pediatric intensive care unit for respiratory failure. The biochemical work-up revealed low vitamin B12 level: $152.6 \mathrm{pg} / \mathrm{ml}(197-866 \mathrm{pg} / \mathrm{ml})$, close to lower limit of folate: $4.62 \mathrm{ng} / \mathrm{ml}(3.1-17.5 \mathrm{ng} / \mathrm{ml})$, increased homocysteine level: $9.85 \mathrm{nmol} / \mathrm{ml}(0-1 \mathrm{nmol} / \mathrm{ml})$, and very low methionine level: $7.32 \mathrm{nmol} / \mathrm{ml}(19-51 \mathrm{nmol} / \mathrm{ml})$. Magnetic resonance imaging of the brain showed white matter changes of the frontal lobes, posterior legs of capsula interna, pons and nucleus dentatus consistent with demyelination. MTHFR deficiency was suspected, and treatment with folinic acid, vitamin B12, methionine and betaine was initiated. The peripheral blood DNA analysis of the patient demonstrated a homozygous mutation of c.1015T>G in MTFHR gene. Both parents were confirmed to be asymptomatic heterozygote carriers. Despite treatment, the prognosis was fatal.

Conclusions As related reports suggest better prognosis with early treatment, pediatricians need to consider MTHFR deficiency in similar cases. Prenatal diagnosis is available and should be encouraged for the future pregnancies. 